\title{
A Scale-Invariant, Machian Theory of Gravitation and Electrodynamics
}

\section{Unified}

\author{
Ram Gopal Vishwakarma* \\ Unidad Académica de Matemáticas, Universidad Autónoma \\ de Zacatecas, C.P. 98068, Zacatecas, ZAC, Mexico
}

\begin{abstract}
As gravitation and electromagnetism are closely analogous long-range interactions, and gravitation is formulated in terms of geometry in general relativity (GR), we expect the latter also to appear through the geometry. This unification has however remained an unfulfilled goal.

The goal is achieved here in a new theory, which results from the principles of equivalence and Mach supplemented with a novel insight that the field tensors in a geometric theory of gravitation and electromagnetism must be traceless, since these long-range interactions are mediated by virtual exchange of massless particles whose mass is expected to be related to the trace of the field tensors. Hence the Riemann tensor, like the analogous electromagnetic field tensor, must be traceless.

Thence emerges a scale- invariant, Machian theory of gravitation and electrodynamics unified, wherein the vanishing of the Ricci tensor appears as a boundary condition. While the field equations of the theory are given by the vanishing divergence of the respective field tensors and their duals, the matter and charge emerge from the spacetime. A quantitative formulation of the emergent fields embodied in 'energymomentum super tensors' follows from the respective Bianchi identities for the two fields. The resulting theory is valid at all scales and explains the observations without invoking the non-baryonic dark matter, dark energy or inflation. Moreover, it answers the questions that the GR-based standard paradigm could not address.
\end{abstract}

Keywords: Gravitation - Fundamental Problems and General Formalism; Electrodynamics; Scale Invariance; Mach Principle; Dark Energy

PACS: 04.20.Cv, 04.20.-q, 98.80.Jk, 95.30.Sf

*Electronic address: vishwa@uaz.edu.mx 


\section{INTRODUCTION}

Gravitation is currently explained through the theory of general relativity (GR). As we know, there are at least two major difficulties with GR. Firstly, it shows an intrinsic difficulty in its unification with the rest of physics. Secondly, it requires ad-hoc epicycles of dark matter and dark energy in order to explain the observations. These 'dark entities' could not be tested so far by any direct detection experiment, besides lacking any convincing theoretical motivation. It appears that the dark sectors have become more like liabilities than assets of GR. Anyway, if a theory requires more than $95 \%$ of the content of the Universe in the form of dark entities, it is an alarming signal to turn back to the very foundations of the theory.

Quantum field theory, on the other hand, is plagued with the divergence difficulties. Though the process of renormalization renders the theory in agreement with experiments (but not solving the problem itself), nevertheless this indicates that we cannot ignore contributions from gravitation at very high energies. It has long been speculated that if gravitation is included, certain infinite sets of divergent Feynman diagrams can give finite results [1].

Thus, we see pressing reasons to have a theory of gravitation compatible with other interactions - perhaps the electromagnetic and quantum aspects may appear emergent in the underlying theory. Expectedly, the properties of the new theory may be beyond the conventional paradigms. In the following, we sketch a geometric scenario wherein gravitation and electrodynamics appear naturally unified. There are promising indications that the quantum theory may also appear from the geometry.

\section{A NOVEL INSIGHT}

Usually in GR, the source matter is described phenomenologically or by singularities. An alternative formulation of the source is developed from a critical review of the following seemingly dissociated topics, which seem to provide a common insight: the conventional formulation of the source fields warrants a revision. This helps to find a Machian formulation of fields purely in terms of geometry, whence emerges a comprehensive theory of gravitation and electrodynamics unified.

(a) A basic requirement of a geometric theory: Since gravitation and electromagnetism are universal long-range interactions, they are expected to be mediated by massless particles. In the latter case, these particles are photons, which are also anticipated from the quantization of electro- 
magnetic radiation predicted by Maxwell's equations. In the same vein then, one prediction of a quantum theory of gravity would be that the interaction would be mediated by the virtual exchange of some massless particles usually called gravitons, which were also predicted by Einstein on the basis of GR. Also, let us recall that in the Lagrangian theory of a relativistic field, the trace of the field tensor is expected to be proportional to the square of the mass of the particle. This is then in a perfect agreement with the vanishing trace of the electromagnetic field tensor $F_{\mu \nu}$ in order to possess the massless mediator - the photon. In the same vein for a massless graviton, the trace $R_{\alpha \beta}$ of the Riemann field tensor $R_{\mu \alpha \nu \beta}$ is expected to vanish, in conformity with the analogous $F_{\mu \nu}$ of electrodynamics. Thus equation

$$
R_{\mu \nu}=0
$$

of the conventional vacuum GR is expected to hold in the form of a simple initial/boundary condition.

\section{(b) Scale-invariance, source-freeness and unifications of gravitation and electrodynamics:}

Since scales are not absolute notions, this symmetry implies that all the coupling constants appearing in a theory must be dimensionless. Let us recall that one of the main obstacles to the construction of a renormalizable quantum field theory of gravity is that the corresponding interaction constant - the Newton's gravitational constant appearing in Einstein's field equation

$$
R_{\mu \nu}-\frac{1}{2} g_{\mu \nu} R_{\sigma}^{\sigma}=-\frac{8 \pi G}{c^{4}} T_{\mu \nu}
$$

- is a dimensional one. It is then possible to think that the scale-invariance may be instrumental in solving problems of quantum divergences and classical singularities in gravitational theories. Thus, one of the most common ways for a particular theory to be renormalizable is that it is scaleinvariant. There is an increasing speculation that a scale-invariant theory may indeed underlie all of physics.

As Maxwell's equations of electrodynamics are scale-invariant, attempts have been made to realize the unification ${ }^{1}$ of gravitation and electrodynamics by enlarging the symmetry group of

\footnotetext{
${ }^{1}$ Despite many efforts by stalwarts like Einstein, Weyl, Kaluza, Schrödinger and Dirac, the unification of electrodynamics with GR has remained an unfulfilled goal. As electrodynamics is also a long-range interaction like gravitation, one would expect the former too to emerge from the spacetime. Considerable efforts have been devoted to geometrize electrodynamics. However, unlike gravitation, it has not so far been possible to make electrodynamics emerge form the spacetime in the form of a purely geometric phenomenon. It may be mentioned that in Einstein-Maxwell theory, electrodynamics is coupled to the geometry (rather than resulting from the geometry)
} 
gravitational spacetime by the inclusion of scale-invariance. Let us note that while being invariant under general coordinate transformations, the Einstein equation (2) is not scale-invariant. The first scale-invariant theory of gravity was due to Weyl, which attempted a unification of electrodynamics and GR by introducing a non-Riemannian geometry [2]. This idea was further modified by Erwin Schrödinger and later revived by Paul Dirac. Although these attempts were largely infelicitous, nevertheless they have spurred an ever-increasing interest in the scale-invariance: a principle which requires the physical laws to be invariant under the scale change of the metric:

$$
g_{\mu \nu} \rightarrow \bar{g}_{\mu \nu}=\omega^{2}\left(x^{\alpha}\right) g_{\mu \nu}
$$

where $\omega\left(x^{\alpha}\right)$ is an arbitrary function of space and time ${ }^{2}$. In this context, it is highly significant to note that the source-free Maxwell equations in 4-dimensions are manifestly scale-invariant [3]. Similarly, the source-free quantum field theory without mass is also scale invariant. These observations perhaps insinuate that a correct geometric theory should be hinged on the seemingly 'source-free' equations, while the source, formulated alternatively, should appear through the geometry. Thus the conventional source representation needs to be revised. A similar implication appears from the gravitational sector too by examining critically the observations in the conventional framework of GR, and also from a critical examination of GR solutions in light of Mach's principle.

(c) Observations versus GR: It is generally believed that GR has been verified by observations/experiments ranging from the solar system to the largest scale - the Universe itself. It would be insightful to scrutinize these tests in order to uncover the underlying implications thereof. One can classify the tests in the following two categories. In the first category, one can include those experiments which do not require any ad-hoc assumption and bolster GR unquestionably. For instance, the category may comprise the experiments performed in the weak gravity of Solar system: the perihelion precession of Mercury's orbit, the deflection of star light by the Sun, the light traveltime delay experiment proposed by Shapiro and the frame-dragging precession of gyroscope (due

\footnotetext{
through the inclusion of a (seemingly) non-geometric element - the electromagnetic tensor - which has to satisfy the Maxwell equations.

${ }^{2}$ As these transformations preserve the angles between vectors (though they shrink or stretch the distances between the two points described by the same coordinate system $x^{\alpha}$ ), this leads to a conservation of the (global) causal structure of the manifold. For this reason, the transformations (3) are called conformal. For $\omega=$ constant, the transformations (3) are called 'scale transformations'. Thus the conformal transformations are localized scale transformations and the scale-invariance is a weaker form of conformal invariance.
} 
to the angular momentum of the rotating earth) measured by the Gravity Probe B experiment. These are supplemented by the observations in the stronger gravitational fields, for instance the observations of the gravitational waves emanated from binary black hole mergers.

In the second category, one can place those cosmological observations which coerce the theory to take refuge in the exotic dark entities - dark matter, dark energy and inflation. The paramount ones in this category are the observations of the supernovae of Type Ia (SNeIa). These are supplemented by several other observations, for instance the observations of the CMB anisotropies, large-scale structure, baryon acoustic oscillations, gravitational lensing, etc. It however appears that the dark sectors have become more like liabilities than assets of GR. While the dark matter has remained elusive to all detection attempts, even a single candidate of dark energy could not have been assigned decisively by particle physics. Worse still, the most favoured candidate of dark energy - the cosmological constant - poses serious conceptual issues, including the notorious uncanny fine tunning problems.

Let us note that while the first category of the observations are explained in terms of the Ricciflat solutions (to be precise, the Schwarzschild and Kerr solutions), i.e., the solutions of the field equation (1), the second category is explained in terms of the Friedmann-Lemaitre-RobertsonWalker (FLRW) solutions of the field equation (2) with the majority of the content of the energystress tensor $T_{\mu \nu}$ in the form of dark entities. An obvious, though superficial, implication of the observations requiring the unconvincing exotic ingredients, is that the field equation (2) of GR may not be valid at the cosmological scales. This has motivated a plethora of modified gravity theories, which though give in to GR at the anvil of Occam's razor.

A radically different and deeper implication of the observations is rooted in the contrived term $T_{\mu \nu}$ that harbours the dark entities: that the conventional representation of the source of gravitation/curvature through $T_{\mu \nu}$ warrants another look. The source-status of $T_{\mu \nu}$ in GR becomes questionable anyway in the presence of various curved solutions obtained in the total absence of $T_{\mu \nu}$. Although the source of curvature in a Ricci-flat solution is conventionally assigned to the singularity present in the solution (which further mars the source-authority of $T_{\mu \nu}$ ), this pathology looses its meaning in the presence of various singularity-free curved solutions of equation (1): for instance, the Ozsváth-Schücking, Taub-NUT and two recently discovered solutions in [4, 5]. Although many other theories, for example Maxwell's theory, possess solutions in the absence of source and do not pose any consistency problem, however, the case of gravitation is different wherein the existence of a source-free solution goes against the requirements of Mach's principle. 
We thus need to revise our ideas about the nature of the interrelation between spacetime and the source matter (i.e., fields in general). If all the (non-trivial) Ricci-flat solutions do possess curvature and the spacetime does ultimately exists whether or not there is $T_{\mu \nu}$ or singularity present, it would be promising to substitute the conventional formulation of source (given in terms of $T_{\mu \nu}$ and singularity) by a more efficient formulation which is applicable universally to all spacetimes. One such formulation is fostered by Mach's principle, as we shall see in the following.

(d) Mach's principle versus GR: Mach's principle ${ }^{3}$, akin to the equivalence principle, was the primary motivation and guiding principle for Einstein in the formulation of GR. Though Einstein stove off Mach's principle in his later years after failing to formulate GR in a fully Machian sense, nevertheless the principle continues to attract a lot of attention due to its aesthetic appeal and enormous impact, and it is widely believed that a viable theory of gravitation must be Machian.

In the absence of a clear statement from Ernst Mach, although there exist a number of formulations of Mach's principle, in essence the principle advocates to shun all vestiges of the unobservable absolute space and time of Newton in favor of the directly observable background matter in the Universe, which determines its geometry and the inertia of an object. Thus the presence of a material background is essential for defining motion and a meaningful spacetime geometry, according to Mach's principle.

Since the principle of covariance (non-existence of a privileged reference frame) results as a consequence of Mach's denial of absolute space, one would expect GR to obey Mach's principle automatically, as Einstein did. To one's surprise however, there are several anti-Machian features in GR [6]. As Mach's principle makes the material background essential for the existence of a spacetime geometry, an isolated object in an otherwise empty Universe should not possess any inertial properties. But this is clearly violated in a class of Ricci-flat solutions, which possess timelike geodesics and a well-defined notion of inertia in the total absence of $T_{\mu \nu}$ or singularity. Further, a global rotation is not allowed by Mach's principle, since the principle does not support an absolute reference frame. Nevertheless, a uniformly rotating Universe does appear in the Gödel solution [7] - a solution of equation (2) obtained in the presence of pressure-less matter and a negative cosmological constant.

It seems that we require radically new ideas and tools to reconcile Mach's principle with grav-

\footnotetext{
3 The name "Mach's principle" was coined by Einstein for the general inspiration that he found in Mach's works on mechanics, even though the principle itself was never formulated succinctly by Mach.
} 
itation. We have perhaps missed the real message the curious failure of this principle in GR wants to convey. Particularly, the presence of the timelike geodesics and a well-defined notion of inertia in the Ricci-flat solutions obtained in the absence of $T_{\mu \nu}$ must not be just coincidental and there must be some source in the spacetime itself. As space is an abstraction from the totality of distance-relations between matter, it appears that not only inertia, but also space and time should emerge from the interaction of matter. That is, the spacetime is not something to which one can ascribe a separate existence, independently of the matter, the former is just an offshoot of the latter. The very existence of the spacetime signifies the presence of the matter (i.e., fields). Thus the existence of spacetime is a necessary and sufficient condition for the existence of fields. This means that the fields are present universally regardless of the geometry of the spacetime.

Thus, we need an alternative formulation of source devised properly from the geometry itself (and not in terms $T_{\mu \nu}$ ) wherein the matter and the geometry occur in one-to-one correspondence. While on one hand, this will render all the Ricci-flat solutions Machian and physically meaningful, on the other hand, it will dispel the unphysical Gödel solution which possesses closed timelike curves and violates causality.

It thus seems that this Machian proposal of defining source through geometry would indeed prove promising. We shall see in the following sections that an energy-momentum tensor of the source fields of gravitation indeed appears purely from the geometry by virtue of the Bianchi identities. In the same vein, the usual energy-momentum tensor of the electromagnetic field also appears in the resulting theory from the symmetries of the spacetime. Moreover, the resulting theory becomes scale-invariant and explains the observations without requiring the inflation, nonbaryonic dark matter and dark energy.

\section{A NEW THEORY WITH $R_{\mu \nu}=0$ AS A BOUNDARY CONDITION}

In an attempt to discover a fundamental geometrical theory of nature, which can integrate in a purely logical fashion the outcome of the above-discussed issues, we assume the following reasonable, a priori postulates:

1. The spacetime of a geometric theory of nature should be 4-dimensional and the standard (pseudo) Riemannian one (in which the covariant derivative of the metric tensor vanishes).

2. Spacetime cannot exist in the absence of matter. 
3. The field tensor of a long-range interaction (which is supposed to be mediated by massless particles) must be traceless.

The theoretical appeal of these naive, self evident and plausible postulates is that they give rise to a new vision of a geometric theory of gravitation and electrodynamics with novel, dramatic implications. The first postulate may be considered as a paraphrase of Einstein's equivalence principle - the only principle that leads to the geometrization of gravitation through the Riemann curvature tensor $R_{\mu \alpha \nu \beta}$. This also indicates that it is the full Riemann tensor that should be instrumental in the geometrization scheme. As the Einstein equation (2) in GR is based on the use of the contracted curvature tensor (i.e. the Ricci tensor $R_{\alpha \beta}$ ), this has led to a certain eclipse of the full Riemann tensor in GR.

The second postulate renders the theory perfectly Machian implying that spacetime itself is field, which is present universally regardless of the geometry of the spacetime. This bestows a dignity of physicality on the spacetime, which otherwise remains an abstract, mind-construct. As the matter field is always accompanied by the ensuing gravitational field and since the latter also gravitates, an important consequence of the adopted postulate is that the geometry of the resulting spacetime should be determined by the net contribution from the two fields. Thus the metric field is entirely governed by considered matter fields, as one should expect from a Machian theory.

The third postulate reveals a cherished feature of a geometric theory of gravitation, which brings it on a par with electrodynamics, through the boundary condition $R_{\mu \nu}=0$. This provides a suitable platform to unify these interactions. By virtue of the condition $R_{\mu \nu}=0$, the Riemann tensor $R_{\nu \rho \sigma}^{\mu}$ reduces to its traceless counterpart - the Weyl tensor $C^{\mu}{ }_{\nu \rho \sigma}$ which is conformally invariant $\left(\bar{C}^{\mu}{ }_{\nu \rho \sigma}=C^{\mu}{ }_{\nu \rho \sigma}\right)$. One can then readily have the vanishing divergence of the Weyl tensor and its dual

$$
\nabla^{\sigma} C_{\mu \nu \rho \sigma}=0, \quad \nabla^{\sigma} * C_{\mu \nu \rho \sigma}=0,
$$

which also results alternatively from the locally scale-invariant Weyl Lagrangian density $\mathcal{L}=$ $\sqrt{-g} C^{\mu \nu \rho \sigma} C_{\mu \nu \rho \sigma}$ by considering the variation with respect to the connection $\delta \mathcal{L} / \delta \Gamma^{\mu}{ }_{\alpha \beta}$. Here $\nabla$ denotes the covariant derivative relative to the metric $g_{\mu \nu}$ and the symbol $*$ denotes the dual operation defined by $* C_{\alpha \beta \mu \nu}=\frac{1}{2} e_{\alpha \beta \rho \sigma} C^{\rho \sigma}{ }_{\mu \nu}$, with $e_{\alpha \beta \gamma \delta}$ representing the Levi-Civita tensor. Equations (4) may be regarded, in the new theory, as the field equations of gravitation analogous to the Maxwellian field equations

$$
\nabla^{\nu} F_{\mu \nu}=0, \quad \nabla^{\nu} * F_{\mu \nu}=0,
$$


strengthening the long-noticed close correspondence between gravitation and electrodynamics. It is well-known that equations (5) result from the locally scale-invariant Lagrangian density $\sqrt{-g} F^{\mu \nu} F_{\mu \nu}$, which is analogous to the Lagrangian density $\sqrt{-g} C^{\mu \nu \rho \sigma} C_{\mu \nu \rho \sigma}$ for gravitation. This deep analogy between gravitation and electrodynamics will make the establishment of the new theory more enlightening, as we shall see in the following. However, unlike the conventional paradigm, equations (5) here represent field in the very presence of source. The source charge indeed appears from the spacetime, as we shall see in section V). While the field equations (4) hold universally by virtue of $R_{\mu \nu}=0$, those in (5) are satisfied by the Killing vector fields present in the considered spacetime (see in section $\mathrm{V}$. Thus gravitation and electrodynamics, both appear reassuringly in the geometric framework of the new theory.

Though, considering equations (4) as the dynamical field equations for gravitation, does not lead to any new physics (since they are trivially satisfied by the Bianchi identities for the Riemann tensor in view of $R_{\mu \nu}=0$ ), however it does have philosophical consequences emphasizing a perfect correspondence between gravitation and electrodynamics. But more than that it ensures that the resulting theory becomes a Yang-Mills type, by virtue of being derivable from the quadratic Lagrangian. (Not surprisingly, it has already been noted that any Ricci-flat solution of the conventional GR is also a solution of the Yang-Mills theory [8].) This is very reassuring as the Yang-Mills theories are known to be renormalizable and corrections are finite at any order of perturbation [9]. Interestingly, the new theory also averts the problems of the usual Yang-Mills theories of gravitation arising from the introduction of matter through the conventional $T_{\mu \nu}$, which forces the theory to be either non-Riemannian or non-reducible to Newton's theory in a weak gravitational field. On the other hand the present Machian theory, wherein the motion of a test particle is expected to be governed by the resultant of the inertial (material) and the gravitational fields, consistently admits Poisson's equation for weak gravity by virtue of the condition $R_{\mu \nu}=0$ [4].

It may be noted that these field equations of gravitation and electrodynamics given in terms of the pure vanishing divergence of the field tensors and their duals, are in fact the Bianchi identities for the respective field tensors written in disguise: while the two equations appearing in (4) are respectively equivalent to

$$
\nabla^{[\kappa} * C^{\alpha \beta] \gamma \delta}=0, \quad \nabla^{[\kappa} C^{\alpha \beta] \gamma \delta}=0
$$

those in (5) are equivalent to

$$
\nabla^{[\kappa} * F^{\alpha \beta]}=0, \quad \nabla^{[\kappa} F^{\alpha \beta]}=0,
$$


where the square bracket [] denotes the antisymmetrized sum over the bracketed indices.

The nomenclature "boundary/initial condition" for equation $R_{\mu \nu}=0$ appears appropriate due to the following. The field equations (4) imply that in order to have $R_{\mu \nu}=0$ in a spatial volume, it is enough to have $R_{\mu \nu}=0$ on its boundary [11]. This justifies our calling $R_{\mu \nu}=0$ as a boundary condition. This terminology is further justified by the fact that $R_{\mu \nu}=0$ appears as a necessary requirement for a geometric formulation of not only gravitation but also electrodynamics, as we shall see in section $\mathrm{V}$.

In comparison to the full Einstein equation (2), the condition $R_{\mu \nu}=0$ is more compatible with the requirements of scale-invariance, i.e. the theory should be free from the dimensional coupling constants. Remarkably, all the Ricci-flat solutions of the conventional GR (which are instrumental for a satisfactory explanation of the observations, as mentioned earlier) are recovered in the new theory, thanks to the condition $R_{\mu \nu}=0$. However, they now have a new Machian feature: all are endowed with fields. Further, any Ricci-flat solution in one metric $g_{\mu \nu}$ is also a solution in the metric $\bar{g}_{\mu \nu}=\omega^{2} g_{\mu \nu}$ for a constant $\omega$, since $\bar{R}_{\mu \nu}=R_{\mu \nu}$ in this case.

Although the proposed scheme of having matter fields in the absence of $T_{\mu \nu}$ may sound surprising and orthogonal to the prevailing perspective, it seems to have many advantages over the conventional approach. The issue then is whether it can be made realistic. That is, if the spacetime constrained by $R_{\mu \nu}=0$ is claimed not to be empty, the solutions of $R_{\mu \nu}=0$ must possess some imprint of the matter present in them. So, do we have any evidence of such imprints in the solutions of $R_{\mu \nu}=0$ ? The answer is, yes.

\section{A. Evidences of 'Emergent Matter’ in Spacetime}

It has been discovered in [4, 5] that it is always possible to write the solutions of $R_{\mu \nu}=0$ in terms of dimension-full parameters, which appear in the Riemann tensor generatively (i.e., when the parameters vanish, the solutions become Minkowskian) and hence, can be attributed to the source of curvature. These parameters can support physical observable quantities such as the mass-energy, momentum or angular momentum or their densities. For instance, the source of curvature in the Schwarzschild solution

$$
d s^{2}=\left(1+\frac{K}{r}\right) c^{2} d t^{2}-\frac{d r^{2}}{(1+K / r)}-r^{2} d \theta^{2}-r^{2} \sin ^{2} \theta d \phi^{2},
$$


can be attributed to the mass $M$ (of the isotropic matter situated at $r=0$ ) through the parameter $K=-2 G M / c^{2}$. Similarly, the dimension-full parameters present in the Taub-NUT solution can be attributed to the mass and the momentum of the source matter, and those in the Kerr solution to the mass and the angular momentum of the source. Though the Kasner solution in its standard form does not contain any dimension-full parameter, it can alternatively be written as [12]

$$
d s^{2}=c^{2} d t^{2}-(1+n t)^{2 p_{1}} d x^{2}-(1+n t)^{2 p_{2}} d y^{2}-(1+n t)^{2 p_{3}} d z^{2}
$$

wherein the dimension-full parameter $n$ can be expressed in terms of the momentum density ${ }^{4} \mathcal{P}$ as $n=\sqrt{G \mathcal{P} / c}$, and the dimensionless parameters $p_{1}, p_{2}, p_{3}$ satisfy

$$
p_{1}+p_{2}+p_{3}=1=p_{1}^{2}+p_{2}^{2}+p_{3}^{2} .
$$

We thus see that the well-known solutions of equations $R_{\mu \nu}=0$ - Schwarzschild, Kerr, Taub-NUT and Kasner - can be written in terms of respectively mass, angular momentum, momentum and momentum density. However if this view is correct, we should also expect solutions of $R_{\mu \nu}=0$, which can support the observables - energy density and angular momentum density. Interestingly, these missing solutions have recently been discovered following this guideline. The first prediction is met in the solution

$$
d s^{2}=\frac{\left(1+4 m z^{2}\right)}{\left(1+m r^{2}\right)^{2}} c^{2} d t^{2}-\frac{d r^{2}}{\left(1+m r^{2}\right)^{4}}-r^{2} d \phi^{2}-\frac{d z^{2}}{\left(1+4 m z^{2}\right)\left(1+m r^{2}\right)^{2}},
$$

discovered in [4], whose curvature is supported by the energy density $\mathcal{E}$ through the parameter $m=G \mathcal{E} / c^{4}$. As solution 111 is curved but singularity-free for all finite values of the coordinates [this is also corroborated by its Kretschmann scalar $=192 m^{2}\left(1+m r^{2}\right)^{6}$, which is well-behaved for all finite values of $r$, it provides, in the absence of any conventional source there, a powerful support to the proposed strategy of representing the source in terms of the dimension-full sourcecarrier parameters, here $m$.

The second prediction is authenticated by the solution

$$
d s^{2}=\left(1-\frac{\ell^{2} x^{2}}{8}\right) c^{2} d t^{2}-d x^{2}-d y^{2}-\left(1+\frac{\ell^{2} x^{2}}{8}\right) d z^{2}+\ell x(c d t-d z) d y+\frac{\ell^{2} x^{2}}{4} c d t d z
$$

\footnotetext{
${ }^{4}$ We cannot expect contributions to the curvature of the homogeneous metric 9 from angular momentum density and energy density. The former is ruled out in the absence of any axisymmetry, like the one present in (12). The latter is ruled out in the absence of any inhomogeneity, like the one present in (11). The positive energy of the homogeneously distributed matter is supposed to be canceled out by the negative gravitational energy at each point (see the discussion towards the end of section VII A.
} 
discovered in [5] wherein the parameter $\ell$ supports the angular momentum density $\mathcal{J}$ via $\ell=$ $G \mathcal{J} / c^{3}$. It may be curious to note that the Kretschmann scalar for 12 is vanishing even for $\ell \neq 0$, though the solution is not Minkowskian then. The solution does not possess any singularity anywhere and strongly supports the conjectured proposal of representing the source in terms of dimension-full source-carrier parameters, here $\ell$. Interestingly, this new solution illuminates the so far obscure source of curvature in the Ozsváth-Schücking solution [13], which would otherwise be in stark contrast with the new proposal of formulating source in terms of the source-carrier parameters, as it does not have any free parameter ${ }^{5}$.

All this must not be mere lucky coincidences and must have some deeper meaning. The presence of the physical measurable quantities such as the energy, momentum, angular momentum and their densities in the solutions of equations $R_{\mu \nu}=0$ ascerts that Fields are ubiquitously present in spacetime without invoking $T_{\mu \nu}$. This would also explain why spacetime bends like a sheet because like sheet, it contains matter (the 'emergent' matter). The symmetry of the considered field distribution, encoded in the metric and Riemann tensors, can single out the correct geometry from the various possible geometris satisfying $R_{\mu \nu}=0$.

One may wonder how the properties of matter can be incorporated into the dynamical equations of the new theory without taking recourse to $T_{\mu \nu}$. This can be achieved by applying the conservation laws and symmetry principles to the new conviction that all spacetimes harbor fields - inertial and gravitational - whose net contribution determines their geometry. For instance, by assuming that the sum of the gravitational and inertial energies in a uniform matter distribution should be vanishing [4, 14], one can obtain the Friedmann equation of the standard 'concordance' cosmology in a kinematic theory (like the Milne model) [15]. This should not be a surprise, as the Friedmann equation, for dust, can also be derived in Newtonian cosmolog by using the continuity equation and the Navier-Stokes equation of fluid dynamics [16].

${ }^{5}$ The Ozsváth-Schücking solution

$$
d s^{2}=-\left(d x_{1}\right)^{2}+4 x_{4} d x_{1} d x_{3}-2 d x_{2} d x_{3}-2\left(x_{4}\right)^{2}\left(d x_{3}\right)^{2}-\left(d x_{4}\right)^{2},
$$

results from 12 by assigning $\ell=2 \sqrt{2}$ and defining new coordinates $x_{1}, x_{2}, x_{3}, x_{4}$ through the transformation $x_{1}=y, \sqrt{2} x_{2}=-(c t+z), \sqrt{2} x_{3}=(c t-z), x_{4}=x$. 


\section{A NEW FORMULATION OF SOURCE}

We have witnessed ample evidence of the presence of fields in the spacetime constrained by $R_{\mu \nu}=0$, which is reassuring for the proposed scheme. Nonetheless, we need to discover, from more fundamental considerations, a concrete mathematical formulation of the source field in purely geometric terms. For this purpose we can proceed as the following.

As the source field is desired to be conserved, let us then ask, "What tensor-like feature of the geometry is automatically conserved?" The answer provides the Bianchi identities as a promising candidate, which is interpreted in terms of the famous maxim: 'The boundary of a boundary is zero' (the boundary of a closed surface, which is already the boundary of a volume, is zero) [17]. The identities can then rightly be expressed in terms of the vanishing divergence of the field tensors, as we have seen in equations (4)-(7). In this context, it would be worthwhile to mention a tensorial formulation of fields proposed by Öktem [18] which emerges purely from the Bianchi identities in any general geometry. This important result can be recapitulated as the following.

Let us consider a tensor antisymmetric in two indices: $H_{\alpha \beta(\mu)}=-H_{\beta \alpha(\mu)}$, where the index in the parenthesis denotes the rest of the indices (if any) of the tensor. Let us assume that the tensor satisfies the Bianchi identities:

$$
3 \nabla_{[\alpha} H_{\beta \gamma](\mu)} \equiv \nabla_{\alpha} H_{\beta \gamma(\mu)}+\nabla_{\beta} H_{\gamma \alpha(\mu)}+\nabla_{\gamma} H_{\alpha \beta(\mu)}=0
$$

Multiplying 14 by $H_{(\nu)}^{\beta \gamma}$, one gets

$$
H_{(\nu)}^{\beta \gamma} \nabla_{\alpha} H_{\beta \gamma(\mu)}-2 H_{(\nu)}^{\beta \gamma} \nabla_{\beta} H_{\alpha \gamma(\mu)}=0
$$

Adding to this the equation obtained from it by the interchange of $(\mu)$ and $(\nu)$, one gets

$$
\begin{aligned}
H_{(\mu)}^{\beta \gamma} \nabla_{\alpha} H_{\beta \gamma(\nu)}+H_{(\nu)}^{\beta \gamma} \nabla_{\alpha} H_{\beta \gamma(\mu)}- & 2\left\{H_{(\mu)}^{\beta \gamma} \nabla_{\beta} H_{\alpha \gamma(\nu)}\right. \\
& \left.+H_{(\nu)}^{\beta \gamma} \nabla_{\beta} H_{\alpha \gamma(\mu)}\right\}=0
\end{aligned}
$$

which can be written in terms of the divergence of a tensor $T_{\alpha \beta(\mu)(\nu)}$ :

$$
\nabla^{\beta} T_{\alpha \beta(\mu)(\nu)}=\frac{1}{2}\left(H_{\alpha \beta(\mu)} J_{(\nu)}^{\beta}+H_{\alpha \beta(\nu)} J_{(\mu)}^{\beta}\right)
$$

where

$$
J_{(\sigma)}^{\beta} \equiv \nabla_{\alpha} H_{(\sigma)}^{\alpha \beta}
$$


and $T_{\alpha \beta(\mu)(\nu)}$ is defined by

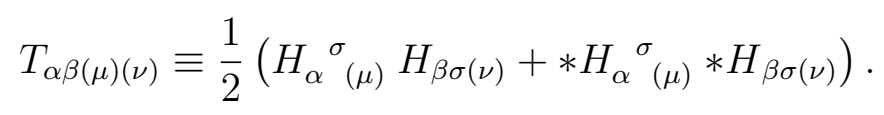

It is remarkable that the tensor $T_{\alpha \beta(\mu)(\nu)}$ given by $(19)$ is defined for any manifold, independently of the matter contents or the field equations and emerges just as a consequence of the geometry by virtue of the Bianchi identities. Let us now come back to our Riemannian spacetime and replace the tensor $H$ with the Riemann (which is now Weyl) tensor by choosing the indices $(\mu)={ }^{\rho}{ }_{\gamma}$, $(\nu)=\rho \delta$; i.e., $H_{\alpha}{ }^{\sigma}(\mu)=C_{\alpha}{ }^{\sigma \rho}{ }_{\gamma}$ and $H_{\beta \sigma(\nu)}=C_{\beta \sigma \rho \delta}$. Interestingly, the definition $(19)$ then provides a covariantly conserved tensor of rank four

$$
T_{\alpha \beta \gamma \delta}=\frac{1}{2}\left(C_{\alpha}{ }^{\sigma \rho}{ }_{\gamma} C_{\beta \sigma \rho \delta}+* C_{\alpha}{ }^{\sigma \rho}{ }_{\gamma} * C_{\beta \sigma \rho \delta}\right),
$$

which is recognized as the Bel-Robinson tensor [11]. Clearly the tensor has a vanishing covariant divergence:

$$
\nabla^{\alpha} T_{\alpha \beta \gamma \delta}=0
$$

since the terms $J_{\rho \delta}^{\beta}$ appearing in 17 amounts, in the present case, to

$$
J_{\rho \delta}^{\beta} \equiv \nabla_{\alpha} C_{\rho \delta}^{\alpha \beta}=0,
$$

by virtue of (4). Historically, the Bel-Robinson tensor has been defined in analogy to the energymomentum tensor of the electromagnetic field. This definition however, appears as somewhat ad-hoc. Additionally, it does not provide any deeper reason for the inclusion of the Weyl tensor. The present formulation, on the other hand, is derived from a fundamental conservation principle wherein the appearance of the conserved Weyl tensor fulfills a basic requirement of a geometric theory wherein the spacetime (whose geometry is given by the Weyl tensor) itself acts as a conserved source. The tensor is symmetric and traceless in all pairs of indices.

Remarkably, this tensor is locally scale-invariant in the present theory. One can check that the transformation (3) leaves the tensor 20 invariant under the condition $R_{\mu \nu}=0$. Moreover, the vanishing trace of the tensor indicates that it must result from a locally scale-invariant Lagrangian, because any Lagrangian corresponding to a traceless energy-momentum tensor is invariant under transformations (3).

Interestingly, a rank-2 tensor of Weyl origin derived from (19) [formulated in analogy to the conventional energy-momentum tensor of GR appearing in [2]], vanishes identically. For instance, the tensor $U_{\mu \nu} \equiv C_{\mu}^{\sigma \rho \kappa} C_{\nu \sigma \rho \kappa}+* C_{\mu}{ }^{\sigma \rho \kappa} * C_{\nu \sigma \rho \kappa}$ vanishes identically, as can be checked by calculating the duals. 


\section{A. $T_{\alpha \beta \gamma \delta}$ : An EFFicient RePresentative of Source}

The Bel-Robinson tensor is generally attributed to the energy-momentum of the gravitational field. This however would not be correct in the case where spacetime itself acts as source. Owing to the fact that matter is the primary requirement to generate the gravitational field, and that the very existence of the tensor is controlled by the curvature which itself is expected to be determined by the net field, it must be the 'effective energy-momentum' of the net field - material plus gravitational - that should be represented by this conserved tensor. Thus we may conjecture that the resultant energy-momentum of the material and the gravitational fields $=\eta T_{\alpha \beta \gamma \delta}$, where $\eta$ is some dimensional constant (which is needed to have the correct dimensions of the density of energy-momentum).

Following Bel [11], one can define the 'effective energy density' $W$ and the 'effective Poynting vector' $P^{\alpha}$ for this net field as

$$
W=\eta T_{\alpha \beta \gamma \rho} u^{\alpha} u^{\beta} u^{\gamma} u^{\rho}, \quad P_{\alpha}=\eta h_{\alpha}^{\sigma} T_{\sigma \beta \gamma \rho} u^{\beta} u^{\gamma} u^{\rho}
$$

which are, in principle, physically measurable quantities in the frame of the comoving observer with velocity $u^{\alpha}$. Here $h^{\alpha \sigma}=g^{\alpha \sigma}-u^{\alpha} u^{\sigma}$ is the projection tensor and $u^{\alpha}$ a timelike unit vector. It may be noted that $W$ and $P^{\alpha}$ arise naturally as invariants under spatial duality rotation, in direct analogy to the Maxwell invariants [19]. Like the positivity of the 'effective energy density' $W$, its observer-dependence too appears as a cherished feature, in close conformity with the energy density in the conventional energy-stress tensor $T_{\mu \nu}$.

Though $T_{\alpha \beta \gamma \delta}$ is non-vanishing for all curved solutions of $R_{\mu \nu}=0$, of particular interest is the solution (12), which witnesses the absence of either $T_{\mu \nu}$ or singularity - the sole refuge of source in the conventional GR. Hence the presence of curvature in solution (12) remains unexplained from the conventional wisdom. This riddle is readily solved when one represents source by $T_{\alpha \beta \gamma \delta}$. The tensor is indeed non-vanishing for solution (12):

$$
T_{0000}=-T_{0003}=T_{0033}=-T_{0333}=T_{3333}=\frac{\ell^{4}}{64} .
$$

This justifies our bestowing a field-full dignity on the spacetime (which naturally harbours (20) and (23)). $T_{\alpha \beta \gamma \delta}$ similarly comes out non-zero for all other curved solutions of $R_{\mu \nu}=0$ and vanishes only when the curvature vanishes. This, taken together with its vanishing covariant divergence, puts forward the tensor as a promising representative of the source. We also note that all the curved 
solutions of $R_{\mu \nu}=0$ are either inhomogeneous or/and anisotropic. The curvature disappears only when the spacetime becomes homogeneous and isotropic (for instance, when we solve $R_{\mu \nu}=0$ for the Robertson-Walker metric yielding the Milne solution (44)), which becomes conformally flat and hence the Weyl tensor vanishes). The resultant field in this case vanishes away due to a cancellation between the positive material field and the negative gravitational field (see section VII A). This justifies our attributing $T_{\alpha \beta \gamma \delta}$ to the resultant field - material plus gravitational.

\section{EMERGENT ELECTROMAGNETISM: A REWARD FROM THE NEW MACHIAN STRAT- EGY}

Various deep analogies between electrodynamics and GR have been discovered in the past (a few have also been noted in this paper), which though remain largely surprising and mysterious coincidences. But, what if the electrodynamics also emerges from the geometry? Would it then not explain away these coincidences, and authenticate the proposed theory? We shall see in the following that the Maxwell equations do emerge inherently from the symmetries of the spacetime in the proposed theory. This should not be surprising since electrodynamics relies on conservation laws and symmetry principles.

Let us consider a Killing vector field $A^{\alpha}$ present in the spacetime. Hence it satisfies the Killing equation

$$
\nabla_{\mu} A_{\nu}+\nabla_{\nu} A_{\mu}=0
$$

$A^{\alpha}$ also satisfies the defining equation of the Riemann tensor:

$$
\nabla_{\mu} \nabla_{\nu} A_{\alpha}-\nabla_{\nu} \nabla_{\mu} A_{\alpha}=R_{\mu \nu \alpha}{ }^{\sigma} A_{\sigma}
$$

which is valid in a general case, even when $R_{\mu \nu}=0$ is not imposed. By the use of 25$]$, equation (26) reduces to

$$
\nabla_{\mu} \nabla_{\nu} A_{\alpha}+\nabla_{\nu} \nabla_{\alpha} A_{\mu}=R_{\mu \nu \alpha}{ }^{\sigma} A_{\sigma}
$$

By adding to this equation the one obtained by the swap of indices $(\mu, \nu, \alpha) \rightarrow(\nu, \alpha, \mu)$ and subtracting the one obtained from the swap of indices $(\nu, \alpha, \mu) \rightarrow(\alpha, \mu, \nu)$, one gets

$$
\nabla_{\nu} \nabla_{\alpha} A_{\mu}+R_{\alpha \mu \nu}^{\sigma} A_{\sigma}=0
$$

By contracting over $\nu$ and $\alpha$, this reduces to the following wave equation in the case of $R_{\mu \nu}=0$ :

$$
\nabla^{\alpha} \nabla_{\alpha} A_{\mu}=0
$$


This in fact leads to the Maxwell equations in the Lorenz gauge [the Lorenz gauge condition $\nabla^{\sigma} A_{\sigma}=0$ follows immediately from $[25$ by contracting over $\mu$ and $\nu$ ]. This can be checked by defining an antisymmetric tensor $F_{\mu \nu}$ by

$$
F_{\mu \nu}=\kappa\left(\nabla_{\mu} A_{\nu}-\nabla_{\nu} A_{\mu}\right) \Rightarrow \nabla^{[\nu} F^{\alpha \beta]}=0 \Rightarrow \nabla^{\mu} * F_{\mu \nu}=0
$$

where $\kappa$ is a (dimensional) constant. By the use of $(25)$, one can then write

$$
\nabla^{\mu} F_{\mu \nu}=\kappa \nabla^{\mu}\left(\nabla_{\mu} A_{\nu}+\nabla_{\mu} A_{\nu}\right)=2 \kappa \nabla^{\mu} \nabla_{\mu} A_{\nu}=0
$$

by virtue of (29), thereby recovering the Maxwell equations (5) with the above-defined $F_{\mu \nu}$ serving as the electromagnetic field tensor. Thus any Killing vector field ${ }^{6}$ present in a Ricci-flat spacetime, gives rise to a solution of Maxwell equations [20]. This is altogether different from the conventional 'electrovac' solutions of Einstein-Maxwell theory [4, 21] wherein the electromagnetic field is coupled to the geometry (rather than emerging from the geometry) by replacing $T_{\mu \nu}$ in (2) with the energy-momentum tensor of the electromagnetic field $E^{\mu \nu}$. Let us note that this tensor, in the present theory, arises naturally from the geometry by putting $H=F$ and dropping the parenthetical indices in the Öktem result 19$)$ :

$$
E_{\alpha \beta}=\frac{1}{2}\left(F_{\alpha}{ }^{\sigma} F_{\beta \sigma}+* F_{\alpha}{ }^{\sigma} * F_{\beta \sigma}\right),
$$

which is divergence-free (as can be checked by applying (31) to the definitions (17) and (18)), symmetric and trace-free like its gravitational counterpart $T_{\alpha \beta \gamma \delta}$. No wonder this tensor results from the earlier-mentioned conformally invariant Lagrangian density, viz. $\sqrt{-g} F^{\mu \nu} F_{\mu \nu}$.

Although equation (31) is recognized as the 'source-free' Maxwell equation", it however appears that the source charge too exists in the geometry with the 'charge carrier' emergent matter.

\footnotetext{
${ }^{6}$ Interestingly, the antisymmetric tensor $F_{\mu \nu}$ defined by $F_{\mu \nu} \equiv \nabla_{\mu} A_{\nu}-\nabla_{\nu} A_{\mu}$ admits an identity $\nabla^{\nu} \nabla^{\mu} F_{\mu \nu}=0$ for an arbitrary $A^{\mu}$ (not necessarily a Killing vector field) in an arbitrary spacetime (not necessarily a Ricci-flat one). This identity has an obvious solution $\nabla^{\mu} F_{\mu \nu}=$ constant, which may give an impression that $\nabla^{\mu} F_{\mu \nu}=0$ is satisfied for an arbitrary vector field $A^{\mu}$ in an arbitrary geometry. However, this is not correct. In fact, assigning the constant in the above solution to zero is equivalent to putting an extra condition on $A^{\mu}$ :

$$
\nabla^{\mu} F_{\mu \nu}=\nabla^{\mu} \nabla_{\mu} A_{\nu}-\nabla^{\mu} \nabla_{\nu} A_{\mu}=0
$$

This is though readily satisfied by virtue of 290 when $A^{\mu}$ is a Killing vector field in a Ricci-flat spacetime.

7 The conventional 'source-full' Maxwell equations are: $\nabla^{\mu} * F_{\mu \nu}=0, \nabla_{\mu} F^{\mu \nu}=j^{\nu}$, where $j^{\nu}$ is the source four-vector. This however shows a discrepancy in the presence of a non-zero $j^{\nu}$ : the dual of $F^{\mu \nu}$ is conserved (covariantly) but $F^{\mu \nu}$ itself is not! This appears at odd with the fact that both these tensors carry exactly the same information - one is obtained from the other by just an interchange of the electric and magnetic fields. Physical,
} 
This is corroborated by the presence of non-vanishing $F_{\mu \nu}$ and $E_{\mu \nu}$, in not only the local situations (for instance, those given by the Schwarzschild and Kerr solutions, which represent the spacetime outside the source matter), but also the cosmological ones wherein there is no 'outside' to 'hide' the source charge through any conventional formulation.

A. Evidences of the Presence of Electromagnetic Source Without Invoking the USUAL SOURCE-REPRESENTATION

EXAMPLE 1: In order to exemplify this, let us first consider the flat Minkowski spacetime

$$
d s^{2}=c^{2} d t^{2}-d x^{2}-d y^{2}-d z^{2}
$$

which is conventionally regarded to represent a completely empty Universe in the absence of $T_{\mu \nu}$ or any singularity. In the present theory however (wherein field is ubiquitous and a completely empty Universe is nonexistent), its vanishing curvature is explained in terms of a cancellation of the uniformly distributed (positive) material field with the uniform (negative) gravitational field. (We shall see in section VII A that this spacetime represents a cosmological solution in the present theory and appears dynamic in suitably chosen coordinates.)

The purpose of considering the example (33) is twofold: It aims to show that (i) matter is ubiquitous which is present even in the Minkowski spacetime, (ii) charge, akin to the charge-carrier matter, is also present in the spacetime. If solution (33) can admit a non-vanishing electromagnetic field, it would ascertain the presence of charge and hence the charge-carrier matter in the very spacetime fabric. We shall see in the following that the solution indeed admits a non-zero electromagnetic field resulting from the spacetime symmetries.

It is well-known that the Minkowski spacetime represents a maximally symmetric spacetime possessing the maximum possible number (ten) of Killing vector fields corresponding to the following symmetries:

(i) four translations generated by the vectors: $\frac{\partial x^{\alpha}}{c \partial t}, \frac{\partial x^{\alpha}}{\partial x}, \frac{\partial x^{\alpha}}{\partial y}, \frac{\partial x^{\alpha}}{\partial z}$ (where $x^{0} \equiv c t, x^{1} \equiv$ $\left.x, x^{2} \equiv y, x^{3} \equiv z\right)$

observable quantities are expected to be conserved. In a similar vein, the conventional GR shows a discrepancy between the Riemann tensor and its dual: i.e., the (double) dual of the Riemann tensor conserves (covariantly), but the Riemann tensor itself does not, unless the conventional source representative $T_{\mu \nu}$ is absent. These discrepancies however disappear in the present theory thereby annunciating that the source must appear through the geometry in a covariant relativistic theory. 
(ii) three spatial rotations generated by the vectors: $(0,0, z,-y),(0,-z, 0, x)$, $(0, y,-x, 0)$

(iii) and three boosts generated by the vectors: $(-x,-c t, 0,0),(-y, 0,-c t, 0)$, $(-z, 0,0,-c t)$.

(The vectors are shown in terms of their contravariant components.) It is now easy to calculate the electromagnetic field corresponding to these Killing vectors in the present case wherein the definition (30) reduces to $F_{\mu \nu}=2 \kappa \nabla_{\mu} A_{\nu}=2 \kappa \partial_{\mu} A_{\nu}$. While the translation vectors (being constant vectors) do not contribute to the field, the rest six Killing vectors lead to the following non-vanishing components of the respective $F_{\mu \nu}$.

Rotation 1: $F_{23}=-F_{32}=2 \kappa$,

Rotation 2: $F_{31}=-F_{13}=2 \kappa$,

Rotation 3: $F_{12}=-F_{21}=2 \kappa$,

Boost 1: $F_{01}=-F_{10}=2 \kappa$,

Boost 2: $F_{02}=-F_{20}=2 \kappa$,

Boost 3: $F_{03}=-F_{30}=2 \kappa$.

As equations (30) and (31) are linear, the principle of superposition holds and the above six solutions sum to a resultant field given by

$$
F_{\mu \nu}=2 \kappa\left[\begin{array}{cccc}
0 & 1 & 1 & 1 \\
-1 & 0 & 1 & -1 \\
-1 & -1 & 0 & 1 \\
-1 & 1 & -1 & 0
\end{array}\right], \quad * F_{\mu \nu}=2 \kappa\left[\begin{array}{cccc}
0 & 1 & 1 & 1 \\
-1 & 0 & -1 & 1 \\
-1 & 1 & 0 & -1 \\
-1 & -1 & 1 & 0
\end{array}\right]
$$

We can now calculate the corresponding electric and magnetic field vectors $E_{\mu}, B_{\mu}$. In the rest frame of an observer $u^{\alpha}$, these vectors are defined as

$$
E_{\mu}=F_{\mu \nu} u^{\nu} \text { and } B_{\mu}=-* F_{\mu \nu} u^{\nu}
$$

Hence by an observer co-moving with the matter, i.e. $u^{\alpha}=(1,0,0,0)$, these vectors are measured as

$$
E_{i}=F_{i 0}, \quad B_{i}=-* F_{i 0}, \quad(i=1,2,3),
$$


implying that

$$
\begin{gathered}
E_{x}=E_{y}=E_{z}=-2 \kappa, \\
B_{x}=B_{y}=B_{z}=2 \kappa .
\end{gathered}
$$

Thus the spacetime (33) possesses a (non-vanishing) constant electromagnetic field, as is expected from a uniform distribution of (charged) matter. As this happens without including into the field equations any separate formulation of source (either charge density or mass density), it shows that the source charge, akin to the neutral matter, does emerge from the geometry (from the symmetries of the spacetime). This corroborates our observation that the spacetime is not different from the fields.

EXAMPLE 2: In order to show that the emergence of the electromagnetic field in the above example is not due to the extreme simplicity of the flat Minkowskian spacetime, and also to solidify our claims further, let us consider a curved spacetime, for instance the Schwarzschild one given by solution $(8)$. Since the metric potentials in this solution do not depend on $t$ or $\phi$, the solution has

$$
\underset{(1)}{A^{\mu}}=\frac{\partial x^{\mu}}{c \partial t}, \quad \underset{(2)}{A^{\mu}}=\frac{\partial x^{\mu}}{\partial \phi} \quad\left(x^{0} \equiv c t, x^{1} \equiv r, x^{2} \equiv \theta, x^{3} \equiv \phi\right) .
$$

as two Killing vectors. The spherical symmetry in $(8)$ implies the existence of two additional Killing vectors

$$
\left.\begin{array}{l}
A_{(3)}^{A^{\sigma}} \frac{\partial x^{\mu}}{\partial x^{\sigma}}=\sin \phi \frac{\partial x^{\mu}}{\partial \theta}+\cot \theta \cos \phi \frac{\partial x^{\mu}}{\partial \phi}, \\
\underset{(4)}{A^{\sigma}} \frac{\partial x^{\mu}}{\partial x^{\sigma}}=-\cos \phi \frac{\partial x^{\mu}}{\partial \theta}+\cot \theta \sin \phi \frac{\partial x^{\mu}}{\partial \phi} .
\end{array}\right\}
$$

In order to find out $F_{\mu \nu}$ resulting from the contributions of all the Killing vectors, one can either follow the procedure followed in Example 1 above, or equivalently can first calculate the 'resultant' Killing field and then calculate the resulting $F_{\mu \nu}$. Following the latter route, the 'resultant' Killing field is obtained as

$$
A^{\mu}=(1,0, \sin \phi-\cos \phi, 1+\cot \theta \cos \phi+\cot \theta \sin \phi)
$$

by summing the four Killing vectors given in (38/39) and remembering that the sum of the Killing vectors is also a Killing vector. $F_{\mu \nu}=2 \kappa \nabla_{\mu} A_{\nu}$ then gives the following non-vanishing indepen- 
dent components of $F_{\mu \nu}$ and its dual:

$$
\left.\begin{array}{l}
F_{01}=\frac{\kappa K}{r^{2}}, \\
F_{12}=-2 \kappa r(\sin \phi-\cos \phi), \\
F_{13}=-2 \kappa r\left[\sin \theta \cos \theta(\sin \phi+\cos \phi)+\sin ^{2} \theta\right], \\
F_{23}=2 \kappa r^{2}\left[\sin ^{2} \theta(\sin \phi+\cos \phi)-\sin \theta \cos \theta\right] ;
\end{array}\right\}
$$

The corresponding electric and magnetic field vectors measured in the static orthonormal frame $(1,0,0,0)$ are given by equations $(36)$. Interestingly, this gives the only non-vanishing component of the electric field as $E_{r}=-\kappa K / r^{2}$ in the present case. The resemblance of this with the Coulomb field of a spherical mass placed at the origin is striking, and asserts that the total charge of this mass must be included in the constant $\kappa$. This provides another, and perhaps stronger, evidence that the charge, and hence the charge carrier matter too, do exist in the spacetime.

The embracement of electrodynamics in the spacetime could be shown here only in the presence of symmetries. Though a formulation in the most general case is desirable, the proposed formulation works in practical situations where we always consider a spacetime with some symmetries, recalling that the relativistic equations become insurmountable in the general case without any symmetries.

\section{ELECTROMAGNETIC WAVES VERSUS GRAVITATIONAL WAVES}

Various cases of a close correspondence between gravitation and electrodynamics have been noted earlier. This correspondence between the two interactions can further be extended in the realm of the corresponding wave equations.

As electrodynamics predicts the existence of electromagnetic waves, gravitation similarly predicts the existence of gravitational waves. Wave propagation of field disturbances becomes inevitable due to the existence of a finite velocity of the propagation of information. It is however believed that, unlike the Maxwell field equations, the Einstein field equation (2) of GR can clearly 
define the waves only in the linearized version of the theory. Equation (2) in the most general case, does not obey the wave equation or reduce to wave-like solutions. This has been the origin of a prolonged debate over the existence of the gravitational waves: whether a particular wave-like solution is a real physical effect or just a coordinate effect.

However, after the direct detection of the gravitational waves, there remains no doubt that the gravitational waves too exist. Hence the lack of a unanimous definition resulting from the field equations warrants immediate attention. In this view, it would be worth-mentioning that a Ricciflat spacetime indeed admits a homogeneous wave equation for the Lanczos tensor $L_{\mu \nu \sigma}$ [22]:

$$
\nabla^{\alpha} \nabla_{\alpha} L_{\mu \nu \sigma}=0
$$

It would be encouraging to note that this equation also holds in the present theory, by virtue of the condition $R_{\mu \nu}=0$. The perfect conformity of the wave equation (43) with the wave equation for the electromagnetic field given by equation 29 :

$$
\nabla^{\alpha} \nabla_{\alpha} A_{\mu}=0
$$

is remarkable and strengthens the correspondence between two interactions in the new theory. This correspondence is reinforced by the fact that the Lanczos tensor $L_{\mu \nu \sigma}$ serves as the potential for the Weyl tensor, in close analogy with the electromagnetic potential $A_{\mu}$ for the electromagnetic field tensor [23]. Interestingly, this potential tensor $L_{\mu \nu \sigma}$ exist only for the Weyl tensor, and not for the Riemann tensor in general [24]. This strongly supports our conjecture that $R_{\mu \nu}=0$ must be an obligatory requirement for a geometric theory of gravitation, meaning - it is the Weyl tensor which is encoded with the gravitational information and not the Riemann tensor in general. It has also been shown that this potential tensor does not exist in general for dimensions higher than four [25]. Taken together with the facts that the Weyl tensor vanishes identically in dimensions less than four and that the electrodynamics exhibits scale-invariance only in four dimensions, this insinuates that the four-dimensional spacetime is the correct platform for gravitation and electromagnetism to appear through the geometry in a scale-invariant theory.

\section{STATUS OF DARK SECTORS IN THE NEW THEORY}

As mentioned earlier, dark matter, dark energy and inflation are required by the GR-based standard cosmology to explain the cosmological observations. It has been shown, through a number 
of studies, that all the cosmological observations are successfully explained in the Milne model (which appears as a particular solution in the present theory) without requiring the dark sectors. We briefly review the results of this finding in the following. For a detailed information, one is advised to see the cited references.

Dark Energy: Dark energy has been brought around in cosmology particularly by the observations of SNeIa. Let us recall that the standard model of cosmology used to be the canonical Einstein-de Sitter model before the SNeIa observations started coming into light two decades ago. As the SNeIa, in the observations, appear fainter than what is predicted by this model, the problem was explained away by requiring about $70 \%$ of the energy budget of the Universe in terms of Einstein's old cosmological constant $\Lambda$. This effectuates an accelerated expansion and hence a larger distance to the SNeIa for their redshift thereby decreasing their luminosity in the model. Now the scope for the agent causing the accelerated expansion has been broadened in terms of the phenomenological models of negative pressure, generally recognized as 'dark energy' models, though $\Lambda$ remains the most favourite candidate for observations. However, $\Lambda$ harbours problems such as the fine-tunning and coincidence problems, as has been mentioned earlier.

Interestingly, the SNeIa observations are explained successfully [15, 26, 27] (without requiring any dark energy) in terms of the homogeneous, isotropic solution of equations $R_{\mu \nu}=0$, viz.

$$
d s^{2}=c^{2} d t^{2}-c^{2} t^{2}\left(\frac{d r^{2}}{1+r^{2}}+r^{2} d \theta^{2}+r^{2} \sin ^{2} \theta d \phi^{2}\right),
$$

obtained by solving $R_{\mu \nu}=0$ for the Robertson-Walker metric. As solution (44) - which is generally recognized as the Milne solution - is also shared by the present theory, it represents the homogeneous, isotropic Universe in the present theory. Though, this solution is conventionally regarded to represent an empty Universe in the framework of GR, it is not devoid of fields in the present theory, as we have already seen (see also section VII A).

In terms of the coordinates $r, \theta, \phi$ and the cosmic time $t$ (co-moving in an expanding reference frame), the Universe appears dynamic in 44 with the Robertson-Walker scale factor $S$ given by

$$
S=c t
$$

and expands at a constant rate throughout the evolution. Nevertheless, solution 44) can be reduced to the simple Minkowskian form (33) in the locally defined coordinates co-moving in a rigid inertial frame. 
It has already been noticed that the coasting model (44) is consistent with the observations of SNeIa without requiring any dark energy. As early as in 1999, the Supernova Cosmology Project team realized from the analysis of their first-generation of the SNeIa data that the performance of the model (44) is practically identical to that of the best-fit $\Lambda$ CDM model [28]. The same was realized later in [15, 26] (See also [29] in this context) by fitting a newer dataset of SNeIa [30] to model [44). The model was further tested in [27] by the highest redshift SNIa $(z=1.914)$ yet discovered [31] and fared well in the test.

One may wonder how the new paradigm, which does not possess any dark energy (and hence an accelerated expansion), manages to reconcile with the observations. The mystery lies in the special expansion dynamics [45] of the model [6] throughout the evolution. We can check that, unlike the standard cosmology, solution (44) efficiently provides different measures of distances without requiring any input from the matter fields. For example, the luminosity distance $d_{\mathrm{L}}$ of a source of redshift $z$ is given, in the new theory, by

$$
d_{\mathrm{L}}=c H_{0}^{-1}(1+z) \sinh [\ln (1+z)]
$$

where $H_{0}$ represents the present value of the Hubble parameter $H=\dot{S} / S$. As has been shown in Figure 1, the luminosity distance of an object of redshift $z$ in the new cosmology is almost the same as that in the standard cosmology for $z \leq 1.3$. This explains why both models are equally consistent with the SNeIa data wherein the majority of the SNe belong to this range of redshift. However, for $z>1.3$, the new cosmology departs significantly from the standard cosmology, as is clear from the figure. Hence observations of more SNeIa at higher redshifts will be decisive for both paradigms.

As all the candidates of dark energy can be assimilated in the energy-stress tensor $T_{\mu \nu}$, and since the latter is absent from the dynamical equations in the new theory, the dark energy and its associated problems, for instance the cosmological constant problem (which appears due to a conflict between the tensor $T_{\mu \nu}$ in equation (2) and the energy density of vacuum in the quantum field theory) and the coincidence problem, are evaded in the new theory.

Inflation: Inflation is required by the standard cosmology to explain the flatness of the Universe (total energy density of the Universe appearing extremely fine-tunned to its critical value) and the observed uniformity of $\mathrm{CMB}$, which has the same temperature everywhere (except for tiny stochastic fluctuations) even in the regions on opposite sides of the sky which appear to lie outside of each other's causal horizon. This cannot be explained in the standard paradigm in terms of some 


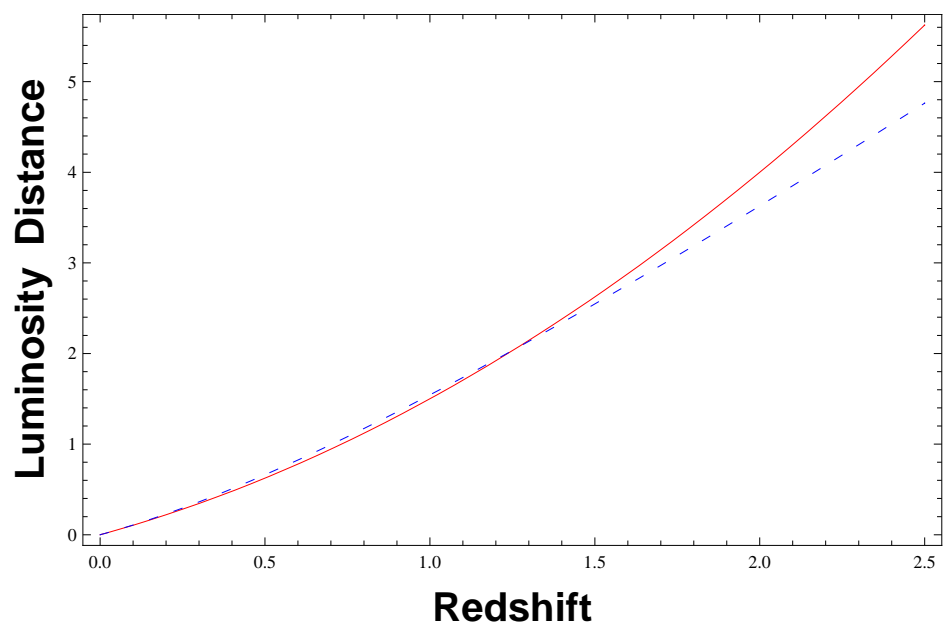

FIG. 1: Luminosity distance in the new paradigm (continuous curve) is compared with that in the $\Lambda$ CDM concordance model $\Omega_{\mathrm{m}}=1-\Omega_{\Lambda}=0.3$ (broken curve). Distances shown on the vertical axis are measured in units of $\mathrm{cH}_{0}^{-1}$.

homogenization process taken place in the baryon-photon plasma operating under the principle of causality, since a finite value for the particle horizon $d_{\mathrm{PH}}(t)=c S(t) \int_{0}^{t} d t^{\prime} / S\left(t^{\prime}\right)$ exists in the standard cosmology.

Inflation is hypothesized to the rescue by claiming that the irregularities in the geometry were evened out by inflation's burst of exponential expansion causing space to become flatter and hence forcing the energy density toward its critical value, no matter what its initial value was. Also, assuming the presence of inflation in the early Universe means the regions that appear to be isolated were actually much closer before inflation than they would have been with only standard Big Bang expansion. Thus, these regions could have been in causal contact prior to inflation and attained a uniform temperature.

However, inflation has its own problems either unsolved or fundamentally unresolvable. There is no consensus on which (if any) inflation model is correct, given that there are many different inflation models. A physical mechanism that could cause the inflation is not known, though there are many speculations. There are also difficulties on how to turn off the inflation once it starts the problem of 'graceful exit'.

The flatness problem is circumvented in the new paradigm due to the absence of $T_{\mu \nu}$ from the dynamical equations. As (45) implies that $d_{\mathrm{PH}}=\infty$ always, thus no horizon exists in the new paradigm and the whole Universe is always causally connected, which explains, in a more natural 
way, the observed overall uniformity of $\mathrm{CMB}$ without invoking the contrived inflationary model [15, 27].

Non-Baryonic Dark Matter: Though a detailed study of the status of dark matter in the new paradigm is still to be made, the problem is expected to be alleviated considerably in the sense that the new paradigm does not require the dark matter, unlike the standard cosmology, to be necessarily non-baryonic. Let us recall that the maximum allowed value of baryon density in the standard cosmology is severely constrained by the theory of nucleosynthesis, which uses the knowledge of the expansion rate and the temperature history of the Universe, along with the details of the nuclear interactions. As a variety of independent estimates of the matter density in the Universe point to a value considerably larger than this limit, the standard cosmology requires the dark matter to be non-baryonic. Since the expansion rate of the Universe in the new paradigm given by (45), does not depend on the matter content, this upper limit for the baryonic matter is not expected to exist in the present theory.

\section{A. On the Milne Solution (44)}

We have seen in the above the usefulness of the homogeneous-isotropic solution of equations $R_{\mu \nu}=0$, viz. (44), which resumes its Minkowskian form in appropriately chosen coordinates and presents a simple model of the Universe in the present theory. It would be encouraging to note that this simple solution not only explains observations without requiring the non-baryonic dark matter, dark energy and inflation, but also avoids some other long-standing problems of the standard cosmology. For instance, the Big Bang singularity and the problem of non-conservation of energy are also averted [4, 6]. This seems to insinuate a deeper insight into the way the Universe must be. The observations perhaps reveal a simpler and more elegant Universe than anyone could have imagined!

One may wonder, "If the Milne model is so useful as appears in the above, why has it failed to attract considerable attention?" The answer is simple - the model is generally believed to represent an unphysical 'empty' universe, which is not quite correct though. Actually there appears to persist a misunderstanding in the literature about the Milne model which is generally portrayed as the 'empty' FLRW model, which is misleading. Although, by considering vanishing energy densities in the FLRW equations of GR, one is led to the same evolution dynamics (44) of the homogeneous, isotropic Universe as that in the Milne model, nevertheless the two models are fundamentally 
different. While the former represents an unphysical empty universe in the framework of GR, the latter is not empty. In fact, the Milne model cannot be recast in the framework of GR. It is a phenomenological model of the Universe, which was developed by Milne independently of GR by assuming the presence of matter in the Minkowskian background. Then from the kinematic relativity and cosmological principle, Milne derived equation (44) as a model of the Universe [15, 32], which is though also shared by the empty FLRW $(k=-1)$ cosmology in GR.

This is, in fact, the origin of the misunderstanding that the empty FLRW model is the Milne model. Nevertheless, in the absence of a concrete theory, the Milne model has remained an adhoc theory and does not give any clue why the presence of matter should not create a possible curvature in the spacetime (if one inquires this in the language of GR). Interestingly, it can be shown as in the following, at least heuristically, that the present theory predicts a vanishing sum of the material and gravitational energies in a uniformly distributed matter expanding/contracting isotropically. Thus the new paradigm, which naturally leads to the Milne model in a cosmological scenario, provides a rigorous theoretical foundation to the Milne model.

In order to show this, let us consider the Kasner solution (9) that represents, in the present theory, a homogeneous distribution of matter expanding/contracting anisotropically. It may be noted from this solution that an isotropic expansion or contraction $\left(p_{1}=p_{2}=p_{3} \neq 0\right)$ from the solution is not possible in general, as $p_{1}, p_{2}, p_{3}$ are constrained by $(10)$. Nevertheless, this readily becomes possible when $n=0$, in which case solution (9) reduces, independently of (10), to solution (33) (which is the same as (44) written in different coordinates). This insinuates that if a homogeneously distributed matter expands or contracts isotropically, its net momentum density vanishes and the resulting spacetime becomes flat. In other words, the energy density of a homogeneously distributed matter does not contribute to the curvature of spacetime. The only way this can happen in the new paradigm is that the positive energy of a uniformly distributed matter be canceled precisely by the negative gravitational energy at each point. Thus the symmetries of homogeneity and isotropy of the Minkowskian spacetime, are responsible for its vanishing curvature. Hawking and Milodinow have also advocated about this cancellation of the material energy by the accompanying negative gravitational energy in the present (homogeneous, isotropic) Universe [14]. 


\section{DISCUSSION AND OUTLOOK}

Gravitation is attributed to the geometry of spacetime. What about the reverse of it? i.e., can any arbitrary spacetime geometry be attributed to gravitation? The executed study in the paper insinuates that it cannot be any arbitrary spacetime, but the one with a trace-free Riemann tensor. In fact, this provides a unified theory of gravitation and electrodynamics. Thus $R_{\mu \nu}=0$ appears as a simple initial/boundary condition (for these interactions to appear through the geometry) and the corresponding Bianchi identities appear as the dynamical field equations of the interactions. This might have a deeper meaning: The long-range character of gravity and electromagnetism implies that the interactions should be mediated by virtual exchange of some massless particles. As the mass of these particles is expected to be related to the trace of the field tensors, the Riemann tensor is expected to be trace-free, in conformity with its electromagnetic analogue - the field tensor $F_{\alpha \beta}$.

A still deeper vision of the constraint $R_{\mu \nu}=0$ now emerges: the source fields should be encoded essentially in the spacetime, and not in the conventional $T_{\mu \nu}$. This is corroborated by a natural derivation of a conserved energy-momentum super tensor $T_{\alpha \beta \gamma \delta}$ from the Riemannian Bianchi identities. Similarly, a conserved energy-momentum tensor $E_{\alpha \beta}$ of the electromagnetic field emerges from the Bianchi identities corresponding to the Killing vector fields. The proposed theory is strongly vindicated by the presence of non-vanishing $E_{\alpha \beta}$ in the cosmological solutions, indicating a universal presence of matter/fields in the spacetime, since charge cannot be expected to exist without the charge carrier matter. This validates the Machian philosophy that the spacetime can exist only in the presence of 'matter'.

Thus the spacetime is not just an empty arena for the struggles of fields and matter particles, but the spacetime continuum itself is a field (gravitational + electromagnetic + material, neutral as well as charged). This is altogether different from the folklore of GR that gravitational field appears through the spacetime, but in order to bring forward its source matter, one has to 'fill' the spacetime with the energy-momentum tensor $T_{\mu \nu}$ (or contrive singularities).

It may be interesting to note that all the tensors appearing in the proposed theory - viz. the tensors Weyl (= Riemann) $C_{\alpha \beta \gamma \delta}$, Bel-Robinson $T_{\alpha \beta \gamma \delta}$, Laczos $L_{\alpha \beta \gamma}$ ('potential' for $C_{\alpha \beta \gamma \delta}$ ), electromagnetic field tensor $F_{\alpha \beta}$ and electromagnetic energy-momentum tensor $E_{\alpha \beta}$ - are all traceless, indicating their origin in scale-invariant Lagrangians. Moreover, they are also covariantly conserved (including the electromagnetic 4-potential $A_{\alpha}$, which appears in the form of a Killing vector field admitted by the spacetime). While the tensors of rank one and two appear to be attributed 
to electromagnetism, those of rank three and four to gravity. Thus it seems possible to reformulate gravity in terms of traceless fields - a typical signature of a scale-invariant theory. This puts forward the proposed theory, which turns out to be a Yang-Mills theory, as a possible platform to embrace the rest two fundamental interactions described by gauge fields which are traceless hermitian.

Although a concrete formulation of fields emerges from pure geometry in the form of tensor $T_{\alpha \beta \gamma \delta}$, which turns out to be the sum of the material and the ensuing gravitational fields (in the spirit of Mach's principle), a formulation of the individual material and gravitational fields is still missing. This may require inputs from other areas allied to gravitation. This formulation is expected to give a vanishing sum of the two fields in the case of a uniform distribution of matter expanding/contracting isotropically.

Though GR was conceived in the spirit of lofty and profound founding principles such as the principles of Mach, equivalence and covariance; its historically developed form appears afflicted by various unwelcome features - for instance, the presence of non-Machian solutions; failure to embrace a covariant formulation of the gravitational energy in the energy-stress tensor; appearance of curved solutions of Einstein field equation in the total absence of $T_{\mu \nu}$ or even singularity (this goes in stark contrast to the founding hypothesis of GR that gravitation manifests itself in terms of the curvature of spacetime whose source is matter which is represented by $T_{\mu \nu}$ or singularity); majority of the solutions turning unphysical; requirement of the epicycles - dark matter, dark energy and inflation in order to explain the observations; and its incompatibility with quantum theory. This has led to believe that GR, in the form as it stands today, may need a revision/replacement. In this view, it would be heartening to note that the proposed theory averts majority of the abovementioned shortcomings of GR and appears more comprehensive while approaching more and more the status envisioned initially by Einstein.

[1] S. Carlip, Rep. Prog. Phys., 64, 885, (2001).

[2] H. Weyl, Sitzungsber. d. Preuss. Akad. d. Wissensch, 465 (1918); H. Weyl, Ann. Phys., Lpz, 59, 101 (1919).

[3] R. Adler, M. Bazin and M. Schiffer, "Introduction to General Relativity", $2^{\text {nd }}$ edition (McGraw-Hill, New York), (1975). 
[4] R. G. Vishwakarma, Int. J. Geom. Methods Mod. Phys., 12, 1550116, (2015).

[5] R. G. Vishwakarma, Pramana J. Phys., 85, 1101, (2015).

[6] R. G. Vishwakarma, Universe, 2, 11, (2016); and the reference therein.

[7] K. Gödel, Rev. Mod. Phys., 21, 447, (1949).

[8] E. E. Fairchild Jr., Phys. Rev. D, 14, 384, (1976).

[9] G. 't Hooft and M. Veltman, Nucl. Phys. B, 44 189, (1972).

[10] A. Einstein, Sitzungber Preuss. Akad. Wiss., 1, 688, (1916).

[11] L. Bel, Gen. Rel. Grav., 32, 2047, (2000) (English translation).

[12] V. V. Narlikar and K. R. Karmarkar, Curr. Sci., 15, 69, (1946).

[13] I. Ozsváth and E. Schücking, An anti-Mach metric, in Recent Developments in General Relativity (Ed. L. Witten), (Pergamon Press, Oxford) 339, (1962).

[14] S. Hawking and L. Milodinow, "The Grand Design”, (Bantom Books, New York), (2010).

[15] R. G. Vishwakarma, Phys. Scripta, 87, 055901, (2013).

[16] J. V. Narlikar, “An Introduction to Cosmology", (Cambridge University Press), (2002).

[17] C.W. Misner, K.S. Thorne and J.A. Wheeler, "Gravitation”, (Freeman, San Francisco), (1973).

[18] F. Öktem, Nuovo Cimento, 58B, 167, (1968).

[19] R. Maartens and B. A. Bassett, Class. Quant. Grav., 15, 705, (1998).

[20] R. M. Wald, "General Relativity", (University of Chicago Press, Chicago), (1984).

[21] C. W. Misner and J. A. Wheeler, Ann. Phys., 2, 525, (1957).

[22] P. Dolan and C. W. Kim, Proc. Rol. Soc. A, 447, 557, (1994).

[23] C. Lanczos, Rev. Mod. Phys., 34, 379, (1962).

[24] E. Massa and E. Pagani, Gen. Rel. Grav., 16, 805, (1984); S. B. Edgar, Gen. Rel. Grav., 26, 329 (1994).

[25] S. B. Edgar and A. Hoglund, Gen.Rel.Grav., 32, 2307, (2000).

[26] R. G. Vishwakarma, Open Astronomy Journal, 6, 14 (2013); Res. Astron. Astrophys., 13, 1409 (2013).

[27] R. G. Vishwakarma, Frontiers of Physics, 9, 98, (2014).

[28] S. Perlmutter, et al., Astrophys. J., 517, 565, (1999).

[29] R. G. Vishwakarma and J. V. Narlikar, Res. Astron. Astrophys., 10, 1195, (2010); Universe, 4, 73, (2018).

[30] A. Riess, et al., Astrophys. J., 659, 98, (2007).

[31] D. O. Jones, et al., Astrophys. J., 768, 166, (2013).

[32] H. Bondi, "Cosmology” (Cambridge University Press, second edition), (1968). 